\title{
The Maize Lethal Leaf Spot 1 Mutant Has Elevated Resistance to Fungal Infection at the Leaf Epidermis
}

\author{
Carl Simmons, ${ }^{1}$ Sabine Hantke, ${ }^{1}$ Susan Grant, ${ }^{1}$ Gurmukh S. Johal, ${ }^{2}$ and Steven P. Briggs ${ }^{1}$ \\ ${ }^{1}$ Pioneer Hi-Bred International, Inc., 7300 N.W. 62nd Avenue, Johnston, IA 50131, U.S.A.; ${ }^{2}$ Department of \\ Agronomy, University of Missouri, Columbia 65211, U.S.A. \\ Accepted 14 July 1998.
}

\begin{abstract}
The maize lethal leaf spot $\underline{1}$ (lls1) mutant exhibits enhanced resistance to fungal pathogens. The $l l s 1$ resistance to Cochliobolus heterostrophus has two components: (i) lesion number is reduced $40 \%$ relative to wild type; and (ii) the lesions that do form often do not contain viable fungus. This lesion sterility is dependent upon leaf maturity and light, whereas reduced lesion number is not. The lls1 lesions express pathogenesis-related proteins at high levels, so lesion sterility likely results from activation of defense systems and necrosis. Reduced lesion number is correlated with a reduction of $C$. heterostrophus spore germination, hyphal growth, and haustoria formation on the leaf epidermis. The rust pathogen Puccinia sorghi has reduced pustule formation on $l l s 1$, and its germination and growth are also slowed on the epidermis. However, after entering the mesophyll through stomata, $P$. sorghi can form pustules on $l l s 1$, and even green islands within necrotic $l l s 1$ lesions. In situ mRNA hybridization shows that Lls 1 is predominantly expressed in the leaf epidermis, coincident with the site of resistance in the mutant.
\end{abstract}

Additional keywords: chitinase, Cochliobolus carbonum, Colletotrichum graminicola, PR1.

The maize lethal leaf spot 1 (llsl) mutation causes the formation of large, spreading, necrotic lesions that eventually consume the leaves and kill the plant. The $l l s 1$ mutation was first described as a single recessive mutation with lesions mimicking those caused by the pathogen Helminthosporium carbonum race 1 (Ullstrup and Troyer 1967). The Llsl gene was later mapped to the short arm of chromosome 1 (Hoisington 1985). The lls 1 lesion formation is developmentally controlled, with older leaf tissue succumbing first (Ullstrup and Troyer 1967), but $l l s 1$ lesion formation is also influenced by light (Close et al. 1995), and by stresses such as wounding (G. Johal, unpublished), necrotic lesion mimic backgrounds (Johal et al. 1994), and pathogen attack (Obanni et al. 1994). Unlike many reported maize lesion mimics, the lls 1 mimic is recessive and its lesion size is unconfined (Walbot et al. 1983; Obanni et al. 1994). Consequently, the LLS1 gene product is viewed as a suppresser of cell death.

Corresponding author: Carl Simmons, Pioneer Hi-Bred International, Inc., 7300 N.W. 62nd Avenue, Johnston, IA 50131, U.S.A.

Telephone: 1-(515) 270-5949; Fax: 1-(515) 270-3367

E-mail: simmonscr@phibred.com
Mutant sector studies indicate that this suppression is cell autonomous (Johal et al. 1994).

The Lls1 gene has been cloned and identified by mutator transposon tagging (Gray et al. 1997). The gene encodes a novel protein with two conserved motifs resembling those found in aromatic ring-hydroxylating (ARH) dioxygenases. By this structural homology it was proposed that LLS1 functions to cleave the ring structure of a phenolic compound. Such a function for the gene product has not been directly demonstrated; however, levels of phenolic compounds are altered in $l l s 1$ plants (Obanni et al. 1994). Phenolic compounds are widespread in plants and are associated with many functions, including stress responses (Nicholson and Hammerschmidt 1992; Dixon and Paiva 1995). In oxidative conditions many phenolic compounds can become highly reactive due to their production of free radicals and reactive oxygen species (ROS). In plants such oxidative conditions can occur during photosynthesis (Asada 1992) and stresses such as wounding and pathogen attack (Tenhaken et al. 1995; Low and Merida 1996). Free radicals and ROS are often associated with and may cause cell death in plants and other organisms (Hockenbery et al. 1993; Kane et al. 1993; Hammond-Kosack and Jones 1996; Jabs et al. 1996). LLS1 might suppress cell death by removing phenolics that are prone to radicalization (Gray et al. 1997).

The $l l s 1$ mutant belongs to a large group of plant mutants classified as lesion mimics. While most of the reported maize lesion mimics are dominant (Johal et al. 1995), many in other species are recessive (Wolter et al. 1993; Dietrich et al. 1994; Greenberg and Ausubel 1993). Lesion mimicry may result from improper production of signals or cell-damaging effects associated with pathogen attack (Walbot et al. 1983), alterations of disease resistance (R) genes (Pryor 1987; Hu et al. 1996), or defects in programmed cell death pathways, with recessive lesion mimics being caused by mutations in genes that encode suppressers of cell death (Johal et al. 1995; Dangl et al. 1996). Lesion mimics may also result from physiological disturbances that cause necrosis or discoloration of the plant, and may not normally be associated with plant pathogen defense or programmed cell death. For instance, the ectopic expression of invertase in tobacco causes both lesion mimicry and systemic acquired resistance (Herbers et al. 1996), even though invertase is not thought to normally play a direct role in defense.

The pathology of relatively few lesion mimics has been investigated in detail. Some are associated with enhanced disease resistance, including elevated pathogenesis-related (PR) 
protein levels and other markers indicative of defense system activation (Wolter et al. 1993; Dietrich et al. 1994). We report that the $l l s l$ lesion mimic displays enhanced resistance to fungal pathogens at the leaf epidermis, where the wild-type Lls1 gene is normally expressed.

\section{RESULTS}

\section{Enhanced resistance to Cochliobolus heterostrophus.}

Inoculation of lls 1 mutant plant leaves with Cochliobolus heterostrophus causes formation of large, necrotic lesions identical in appearance to those that form spontaneously on lls1 (Fig. 1). In this report, such lesions will be referred to as lls1-type lesions whether or not they occur spontaneously or in response to pathogen inoculation. On control lls 1 plants inoculated with a blank Tween 20 solution, there is no induction of these $l l s 1$-type lesions relative to uninoculated plants, although a few spontaneous $l l s 1$-type lesions often develop at the leaf tip as part of the normal progression of llsl lesion formation upon aging (Table 1 , rows $\mathrm{G}-\mathrm{J}$ ).

Inoculation with $C$. heterostrophus also results in reduced lesion numbers on $l l s 1$ plants relative to wild-type siblings (Fig. 1). This reduction averages about $40 \%$, with individual experiments ranging from 37 to $61 \%$ (Table 1, rows A-F). Reduced lesion numbers on $l l s 1$ occurred on all leaves tested, leaves L4, L5, and L6 (Table 1, rows A-F). The C. heterostrophus-induced $l l s l$-type lesions are often sterile; that is, less than half contain viable fungus recoverable in the carrot agar assay. In contrast, $C$. heterostrophus-induced lesions on wild-type plants are almost always fertile and contain viable fungus (Table 2, experiment A).

The extent of $C$. heterostrophus spore germination, hyphal growth, appressorium formation, and lesion initiation on the leaf surfaces of $l l s 1$ and wild-type sibling plants was determined. On $l l s 1$ plants progression through each of these categories was retarded relative to that on wild-type plants (Table 3 ). The reduced spore germination and germ tube growth were correlated with the reduced number of lesions, which indicates that resistance occurs at the leaf surface.

\section{lls1 enhanced resistance to Puccinia sorghi.}

Inoculation of llsl plants with Puccinia sorghi, the causal agent of common rust, leads to the production of pustules (Fig. 2 ). Such spore production indicates that the $P$. sorghi infection does not necessarily induce sterile $l l s l$-type lesion formation, as does $C$. heterostrophus. During the 7 to 10 days needed for pustules to appear, $l l s 1$ lesions do form, but to about the same extent as they do on uninoculated $l l s 1$ plants during this period. We have not ruled out that $P$. sorghi induces some of these lesions. We have, however, observed that pustules are sometimes at the center of green islands within $l l s l$ necrotic tissue (Fig. 2). This indicates that $P$. sorghi can actually suppress $l l s l$-type lesion formation. Eventually, however, after maturation, rust pustules can become initiation centers for llsl-type necrotic lesions. From infection through to mature spore production, though, $l l s 1$ plants do support $P$. sorghi infection.

There are, nevertheless, fewer pustules formed on $l l s 1$ plants than on wild-type siblings (Table 4). This reduction ranged from 52 to $85 \%$, with an average of $70 \%$. The rust pustules on $l l s l$ do contain distinct spores; however, pustule size is often smaller than on wild type. Taken together, these data indicate that $l l s 1$ resists $P$. sorghi infection, even though successful infections do result in some pustule formation. When spore germination, hyphal growth, and stomatal penetrance were examined, all were moderately slowed on $l l s 1$ plants (Table 3). It appears that growth of $P$. sorghi, like that of $C$. heterostrophus, is retarded at the leaf surface, and that this results in a significant portion of the reduced pustule or lesion number.

\section{Effect of light on resistance.}

The formation of spontaneous lesions on $l l s l$ plants is light dependent (Close et al. 1995), and so the dependence upon light of $l l s 1$ resistance to $C$. heterostrophus was investigated. Plants were inoculated with $C$. heterostrophus and then placed in a dark growth chamber for $48 \mathrm{~h}$. This resulted in a 20 to $59 \%$ reduction (average $=41 \%$ ) in lesion numbers on $l l s 1$ plants relative to wild-type siblings (Table 1 , rows $\mathrm{K}-\mathrm{M}$ ). In a similar experiment, a dark preinoculation treatment for $24 \mathrm{~h}$ was done, and after inoculation the plants were kept in the dark $48 \mathrm{~h}$. The number of lesions was reduced by 32 to $43 \%$ (average $=38 \%)$ on $l l s 1$ plants (Table 1 , rows N-P). In yet another experiment, the plants were kept in the greenhouse, but one set of plants had the sixth leaf wrapped in aluminum foil to block light. The foil was applied $4 \mathrm{~h}$ prior to inoculation. The leaves were inoculated in low light, and then rewrapped with foil and returned to the greenhouse for $48 \mathrm{~h}$, at which time lesion numbers were counted. In this experiment, a $26 \%$ reduction in lesion number was observed under the foil on $l l s 1$ plants relative to wild-type plants (Table 1, row Q). Taken together, these results indicate that the reduced lesion numbers are not dependent upon light, at least $24 \mathrm{~h}$ before or $48 \mathrm{~h}$ after inoculation.

The dependence of $l l s l$ lesion sterility upon light was also investigated. Plants were held in the dark or light for $48 \mathrm{~h}$, then inoculated, and held in the dark for $48 \mathrm{~h}$, at which time samples were taken. The lesions found on $l l s 1$ plants in the light were similar to those that form spontaneously. With dark pretreatment the $l l s 1$ lesions tended to looked like $C$. heterostrophus lesions; however, some appeared partially converted to $l l s$ l-type lesions, but were not yet dried, tan, papery, and necrotic. The lesions on wild-type siblings contained viable fungus 79 to $97 \%$ of the time whether grown in the light or dark, with or without a dark pretreatment (Table 2, experiments A-C). On lls 1 plants, lesions were often sterile when grown in the light ( $42 \%$ lesions fertile) or when given a dark pretreatment but light post-inoculation treatment (43\% lesions fertile). The $l l s 1$ plants given no dark pretreatment, but $48 \mathrm{~h}$ of dark post-inoculation treatment, had fertile lesions to almost the same level as wild-type plants, $77 \%$ versus $90 \%$ (Table 2, experiment B). It is apparent that $l l s l$ lesion sterility is dependent upon light, but that this sterility is largely unaffected by light treatment just prior to inoculation.

\section{Dependence of $l l s 1$ resistance on tissue maturity.}

The formation of spontaneous $l l s l$ lesions is developmental, with the more distal portions of the leaf forming lesions first (Ullstrup and Troyer 1967). There is a tendency for the $l l s 1$ necrosis to progress more rapidly along the margins and midribs of the leaves. When $l l s 1$ plants were inoculated with a necrotrophic pathogen such as $C$. heterostrophus, we observed llsl-type lesions as has been noted (Obanni et al. 1994). Inoculation with $C$. 
heterostrophus helped delineate the zone of competency for $l l s 1$ type lesion formation. When L7 plants are inoculated, L1, L2, and L3 leaves die if they are not already dead. The L4 and L5 leaves form $l l s 1$-type lesions along their entire length. The sixth leaf is usually transitional, with the basal half often showing

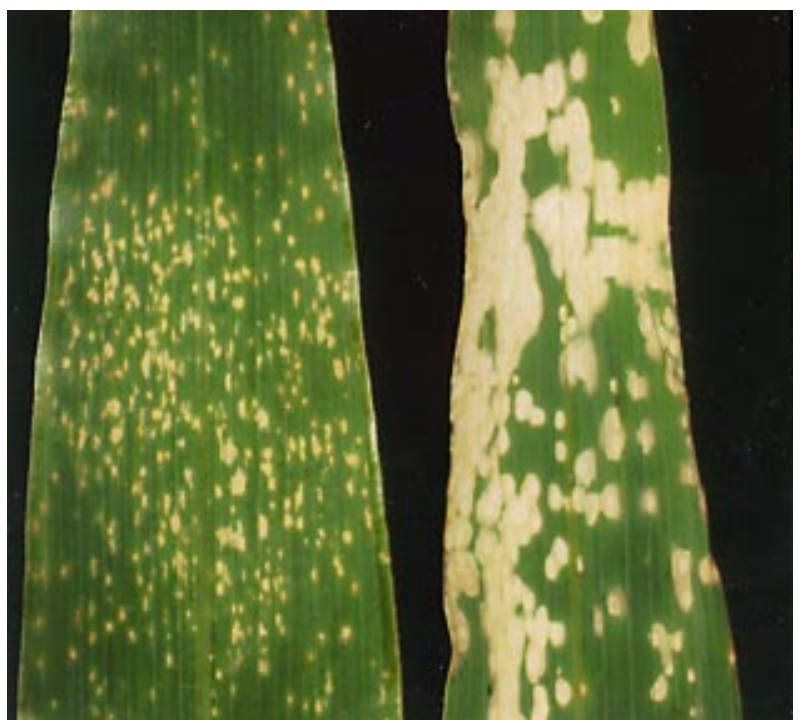

Fig. 1. Wild-type sibling (left) and lethal leaf spot 1 (lls1)-infected (right) leaves were inoculated with Cochliobolus heterostrophus. Two days later they were photographed. There are large necrotic lesions on the $l l s 1$-infected leaf and a reduced number of lesions. normal pathogen-induced lesions, and the upper half showing only $l l s l$-type lesions. The seventh leaf is similarly transitional.

When the leaf whorl containing sixth and seventh leaves is inoculated with $C$. heterostrophus, a patch of $C$. heterostrophus-like lesions forms. When the leaf expands far enough to place this patch at the center of the leaf, the leading lesions begin to convert to $l l s 1$-type lesions (Fig. 3). Leaf explant studies indicate that this transition corresponds to increased lesion sterility (Table 2, experiment D). For a patch of lesions undergoing conversion, the most basal $C$. heterostrophus-like

Table 2. Frequency of viable Cochliobolus heterostrophus recovered from pathogen-induced lesions on wild-type and $l l s 1$ plants

\begin{tabular}{|c|c|c|c|c|}
\hline \multirow{2}{*}{$\begin{array}{l}\text { Ex- } \\
\text { peri- } \\
\text { ment }\end{array}$} & \multirow[b]{2}{*}{ Treatment } & \multicolumn{3}{|c|}{$\begin{array}{l}\text { Lesion explants } \\
\text { with fungus }(\%)\end{array}$} \\
\hline & & $\mathbf{N}^{\mathbf{a}}$ & Avg. & SE \\
\hline \multirow[t]{3}{*}{ A } & Standard inoculation in light $48 \mathrm{~h}$ & & & \\
\hline & Wild-type & 3 & 97 & 2 \\
\hline & lls 1 & 3 & 42 & 17 \\
\hline \multirow[t]{3}{*}{ B } & In light, inoculation, then in dark $48 \mathrm{~h}$ & & & \\
\hline & Wild-type & 3 & 90 & 6 \\
\hline & lls1 & 3 & 77 & 12 \\
\hline \multirow[t]{3}{*}{$\mathrm{C}$} & $48 \mathrm{~h}$ in dark, inoculation, then in light $48 \mathrm{~h}$ & & & \\
\hline & Wild-type & 1 & 79 & - \\
\hline & $l l s 1$ & 1 & 43 & - \\
\hline \multirow[t]{4}{*}{$\mathrm{D}$} & Whorl inoculation in light & & & \\
\hline & Wild type & 2 & 93 & 8 \\
\hline & llsl (wild type-like lesions) & 2 & 75 & 17 \\
\hline & lls1 (lls1-type lesions) & 2 & 30 & 27 \\
\hline
\end{tabular}

${ }^{a}$ Number of separate inoculation experiments from which lesion explants were obtained for the lesion sterility assay. Number of lesion explants used per experiment ranged from 35 to 80 .

Table 1. Lesion numbers resulting from Cochliobolus heterostrophus inoculation of $l l s 1$ and wild-type plants

\begin{tabular}{|c|c|c|c|c|c|c|c|}
\hline \multirow[b]{3}{*}{ Experiment } & \multicolumn{6}{|c|}{ Lesions per leaf ${ }^{\text {a }}$} & \multirow{3}{*}{$\begin{array}{c}\text { Reduction } \\
(\%)\end{array}$} \\
\hline & \multicolumn{3}{|c|}{ Wild type } & \multicolumn{3}{|c|}{ lls1 } & \\
\hline & $\mathbf{N}^{\mathbf{b}}$ & Avg. & SE & $\mathbf{N}$ & Avg. & SE & \\
\hline \multicolumn{8}{|c|}{ Standard inoculations in light } \\
\hline A. Leaf 6 & 3 & 460 & 63 & 5 & 276 & 39 & 40 \\
\hline B. Leaf 6 & 3 & 533 & 88 & 5 & 280 & 1 & 47 \\
\hline C. Leaf 6 & 7 & 472 & 127 & 8 & 296 & 33 & 37 \\
\hline D. Leaf 5 & 3 & 420 & 157 & 5 & 254 & 31 & 40 \\
\hline E. Leaf 5 & 7 & 478 & 72 & 8 & 280 & 87 & 41 \\
\hline F. Leaf 4 & 3 & 316 & 13 & 5 & 120 & 23 & 61 \\
\hline \multicolumn{8}{|l|}{ Control lls 1 plants } \\
\hline G. No inoculation & - & - & - & 4 & 27 & 4 & \\
\hline H. No inoculation & - & - & - & 4 & 18 & 3 & \\
\hline I. Tween 20 blank & - & - & - & 3 & 18 & 4 & \\
\hline J. Tween 20 blank & - & - & - & 3 & 7 & 2 & \\
\hline \multicolumn{8}{|c|}{ Light, then $48 \mathrm{~h}$ post inoculation in dark } \\
\hline $\mathrm{K}$. & 4 & 400 & 61 & 4 & 165 & 47 & 59 \\
\hline $\mathrm{L}$. & 4 & 150 & 40 & 4 & 120 & 35 & 20 \\
\hline M. & 3 & 243 & 110 & 3 & 133 & 15 & 45 \\
\hline \multicolumn{8}{|c|}{$24 \mathrm{~h}$ dark, then $48 \mathrm{~h}$ post inoculation in dark } \\
\hline $\mathrm{N}$. & 7 & 110 & 10 & 5 & 66 & 19 & 40 \\
\hline O. & 7 & 107 & 17 & 5 & 73 & 17 & 32 \\
\hline P. & 3 & 88 & 22 & 3 & 50 & 12 & 43 \\
\hline \multicolumn{8}{|c|}{$4 \mathrm{~h}$ dark, then $48 \mathrm{~h}$ post inoculation in dark (foil) } \\
\hline Q. & 10 & 548 & 116 & 7 & 403 & 47 & 26 \\
\hline \multicolumn{8}{|c|}{ Whorl inoculation in light } \\
\hline $\mathrm{R}$. & 4 & 234 & 55 & 4 & 188 & 21 & 20 \\
\hline S. & 12 & 152 & 18 & 12 & 109 & 16 & 29 \\
\hline $\mathrm{T}$ & 3 & 205 & 25 & 3 & 108 & 28 & 47 \\
\hline
\end{tabular}

${ }^{a}$ Leaf 5 or 6 of seedling plants if not otherwise specified.

${ }^{\mathrm{b}}$ Number of plants used per experiment. 
lesions are $75 \%$ fungal-bearing, whereas the foremost converted lls1-type lesions are only $30 \%$ fungal-bearing. As described above, inoculation of whole leaf blades results in fewer lesions formed on $l l s 1$ throughout the length of the blade. In the whorl inoculation assay we observed a 20 to $47 \%$ reduction (average $=$ $32 \%$ ) in lesion number for the lower portions of leaves 6 and 7 where the lesions are C. heterostrophus-like (Table 1, rows $\mathrm{R}-$ $\mathrm{T})$. This whorl inoculation assay indicates that the lower portion of the sixth and seventh leaves, while not competent to form $l l s 1$ lesions, nonetheless exhibits reduced lesion numbers. Reduced lesion number is thus separable from $l l s l$-type lesion formation in the light by maturity differences. However, the lesion explant study indicates that the lesion sterility is largely dependent upon maturity-based competency to form lls 1 -type lesions. There may be an increased tendency for sterile lesions even in the basal regions, since on $l l s 1$ only $75 \%$ of the basal $C$. heterostrophus-like

Table 3. Cochliobolus heterostrophus and Puccinia sorghi growth on $l l s 1$ and wild-type (wt) leaf surfaces

\begin{tabular}{|c|c|c|c|c|}
\hline \multirow[b]{2}{*}{ Pathogen/growth stage } & \multicolumn{2}{|c|}{ Experiment A } & \multicolumn{2}{|c|}{ Experiment B } \\
\hline & $\%$ wt & $\%$ lls1 & $\% \mathrm{wt}$ & $\%$ lls 1 \\
\hline \multicolumn{5}{|l|}{ C. heterostrophus ${ }^{\mathrm{a}}$} \\
\hline No germination & 0.5 & 4.6 & 1.5 & 14.1 \\
\hline Short hyphae & 2.6 & 11.7 & 4.0 & 12.6 \\
\hline Long hyphae ${ }^{b}$ & 22.9 & 49.8 & 15.5 & 19.7 \\
\hline Appressoria formation & 17.2 & 16.3 & 18.0 & 5.5 \\
\hline Appressoria and lesion & 56.8 & 17.6 & 61.0 & 48.0 \\
\hline \multicolumn{5}{|l|}{ P. sorghic } \\
\hline No germination & 31.5 & 49.5 & 48.0 & 76.0 \\
\hline Hyphae present ${ }^{b}$ & 33.0 & 26.0 & 22.0 & 11.0 \\
\hline Stomatal penetration & 35.5 & 24.5 & 30.0 & 13.0 \\
\hline
\end{tabular}

a Number total spores counted: experiment A, wt $=239$, lls $1=192$; experiment $\mathrm{B}$, wt $=200, l l s 1=127$.

b Short hyphae $<3 \times$ spore length; long hyphae $>3 \times$ spore length. Rust hyphae any length not entered in stomata.

${ }^{\mathrm{c}}$ Number total spores counted: experiment A, wt $=200$, lls $1=200$; experiment $\mathrm{B}$, wt $=100, l l s 1=81$.

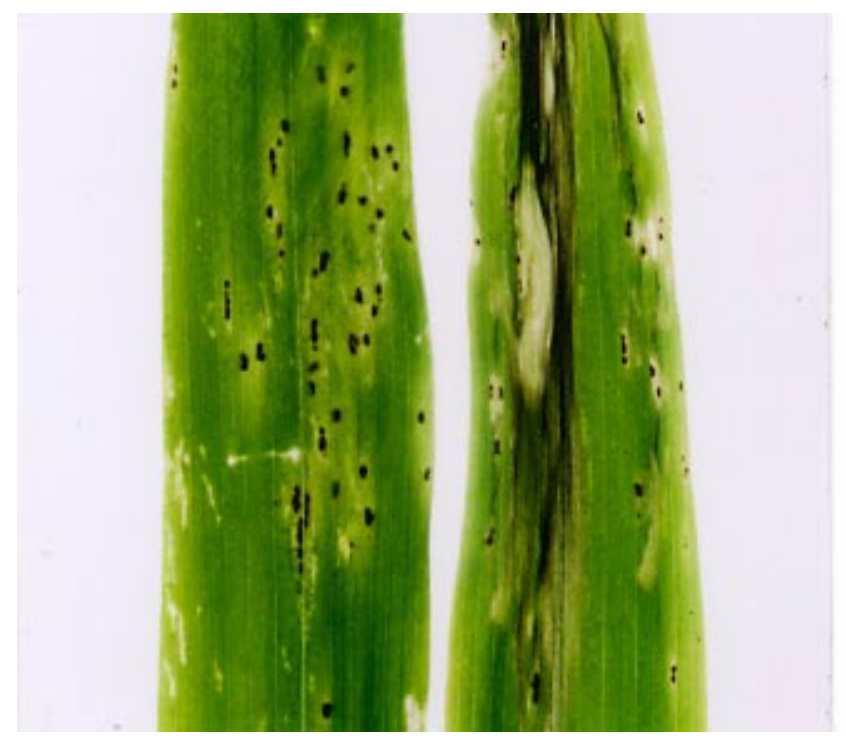

Table 4. Puccinia sorghi rust pustules on leaves of $l l s 1$ and wild-type plants

\begin{tabular}{|c|c|c|c|c|c|c|c|}
\hline \multirow{3}{*}{$\begin{array}{l}\text { Experi- } \\
\text { ment }\end{array}$} & \multicolumn{6}{|c|}{ Pustules per leaf } & \multirow[b]{3}{*}{ Reduction (\%) } \\
\hline & \multicolumn{3}{|c|}{ Wild type } & \multicolumn{3}{|c|}{$l l s 1$} & \\
\hline & $\mathbf{N}^{\mathbf{a}}$ & Avg. & SE & $\mathbf{N}^{\mathrm{a}}$ & Avg. & SE & \\
\hline A & 15 & 48.4 & 8.5 & 21 & 7.3 & 1.6 & 85 \\
\hline B & 8 & 34.6 & 8.2 & 12 & 9.3 & 2.3 & 73 \\
\hline $\mathrm{C}$ & 15 & 25.2 & 6.9 & 21 & 12.0 & 2.5 & 52 \\
\hline $\mathrm{D}$ & 8 & 45.9 & 13.6 & 7 & 13.9 & 6.9 & 70 \\
\hline
\end{tabular}

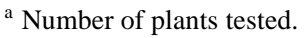
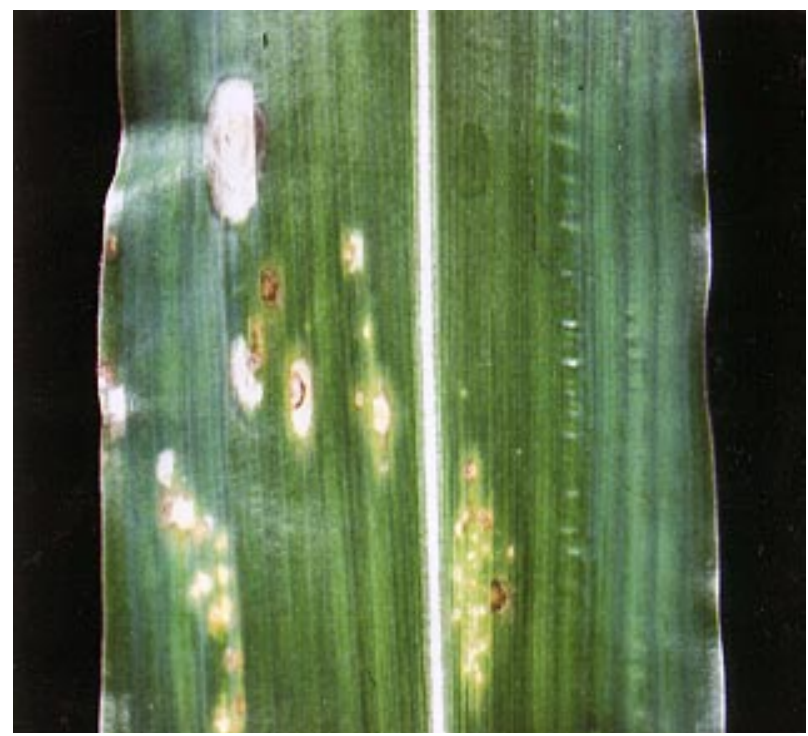

Fig. 3. A cluster of Cochliobolus heterostrophus-induced lesions produced by whorl inoculation of lethal leaf spot 1 (lls 1$)$. Bottom of photo is toward base of leaf; top toward tip. As the lesions cross the middle of the sixth leaf, the leading lesions convert from small orangish $C$. heterostrophus-like lesions to larger, tan lls1-type necrotic lesions.

Fig. 2. Left panel: Rust pustules on wild-type (left) and lethal leaf spot 1 (lls 1 )-infected (right) leaves 8 days after inoculation with Puccinia sorghi. The rust pustules on the $l l s 1$-infected leaf occur without necessarily causing $l l s 1$-type necrosis. Pustules on $l l s 1$-infected plants are often smaller than those on wild-type plants. The $l l s l$-infected plants nonetheless do bear some spontaneous, or possibly $P$. sorghi-induced, lesion mimic necrosis, as indicated here by the large $l l s l$-type lesion stripe on the $l l s l$-infected leaf. Right panel: Rust pustules on $l l s l$-infected plants with green islands surrounded by necrotic lls 1 -type lesion mimic tissue 7 days after inoculation. 
lesions were fertile whereas on wild-type plants $93 \%$ were fertile, but this may be because the basal lesions in the cluster were already partially converted to $l l s l$-type (Table 2 , experiment D).

\section{PR protein levels.}

The pattern of PR protein expression in $l l s 1$ plants was investigated with Western blots (immunoblots) and antibodies against PR1 and chitinase. In an experiment to determine whether $l l s 1$-type lesions accumulate PR proteins, 3-mm-thick concentric rings of leaf tissue emanating from around spontaneously formed $l l s l$ lesions were collected. The Western blots with PR antibodies indicated that $l l s l$-type lesions accumulate high levels of PR1 and chitinase proteins. The gradient of PR1 protein levels extended to at least $1.5 \mathrm{~cm}$ around the lesions (Fig. 4). Whether $l l s l$ plants have constitutively higher resident PR gene expression was also investigated. The primary leaves of three-leaf $l l s l$ seedlings, which did not yet have any lls1-type lesions anywhere on the plants, did not show elevated PR protein levels. In another experiment, a gradient of eight $2.5-\mathrm{cm}$ leaf sections along the length of the leaves revealed that there were low PR1 and chitinase protein levels, except in the tip section containing the $l l s l$-type lesions. This length gradient experiment also indicated that there is not a marked difference in PR1 and chitinase protein levels in the basal portion of the leaf relative to the distal portion that is competent to form $l l s 1$-type lesions. A comparison of $l l s l$ and wild-type plants with either light-grown or dark-grown leaves (28 h wrapped in aluminum foil) did not indicate any change in PR1 and chitinase protein levels (data not shown). In summary, these data indicate that $l l s 1$ plants do not have generally higher levels of PR proteins apart from the zone of high PR protein accumulation surrounding $l l s 1$ lesions.

\section{In situ localization of $L l s 1$ gene expression.}

The Llsl gene expression pattern was investigated by in situ mRNA hybridization. In wild-type leaves the Lls1 transcripts are localized predominantly in the upper and lower epidermis, and in sclerenchyma cells of vascular bundles. All epidermal cells, including silica cells and stomates, strongly express Lls1. Protoxylem elements and bundlesheath cells show an intermediate level of expression, whereas mesophyll cells display a very low level of expression. Lls 1 expression is undetectable in metaxylem, phloem, and companion cells (Fig. 5). There are three types of vascular bundles in the leaf blade of maize: large (including the median vein in the midrib), inter-

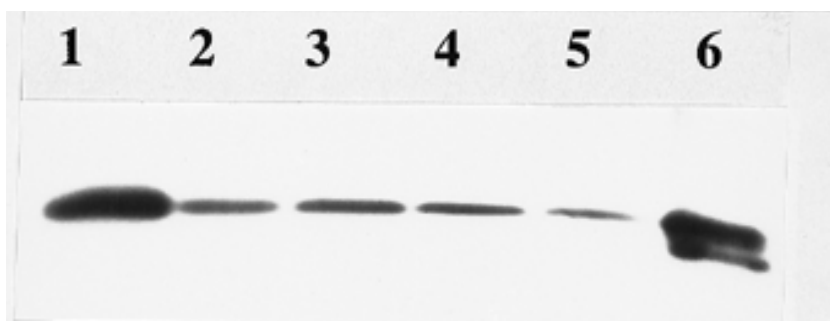

Fig. 4. Ring gradient experiment showing pathogenesis-related (PR) protein expression surrounding spontaneous lethal leaf spot 1 (lls 1)-type lesions. Western blot (immunoblot) probed with anti-PR1b protein antiserum. Lane 1: 0 to $3 \mathrm{~mm}$ of leaf tissue closest to $l l s 1$ lesions and including the lesion margin. Lane 2: tissue 3 to $6 \mathrm{~mm}$ around lesion. Lanes 3-5: tissues 6 to 9,9 to 12 , and 12 to $15 \mathrm{~mm}$ around lesion, respectively. Lane 6: a positive control for maize PR1 expression. mediate, and small. Cell specificity of Lls1 expression is the same in all bundle types. Sclerenchyma strands occur on both adaxial and abaxial surfaces of large and intermediate veins and at the leaf margin along the blade; all of these sclerenchyma strands show strong Lls 1 expression.

The $l l s l$ lesion development is maturity dependent. Lesions develop first on older leaves, and within a leaf the lesions develop first at the tip, which is older than the base. In four-leaf stage V4 seedlings typically 2 to $4 \mathrm{~cm}$ of the leaf 2 tip show $l l s 1$ lesions, the tip of leaf 3 may show a few lesions, while leaf 4 is lesion free. To investigate the possibility that the age dependence of lesion development is correlated with Lls1 gene expression during developmental maturation, the expression pattern of $L l s 1$ was determined in tips of leaves 2 and 4 of wild-type plants at the V4 stage. No differences in Llsl expression pattern or level were detected (data not shown). The same is true for the pattern at the base of leaves 2 and 4, where cells are younger and where at this stage there are no lesions in llsl plants. These results show that Llsl gene transcription is not under significant developmental control between these two plastochrons, even though in mutant plants these leaves encompass the transition to $l l s l$ lesion development. Hence, the age dependence of lesion development is not directly correlated with $l l s 1$ mRNA accumulation in leaves at the V4 stage.

Developmental control of the Lls1 gene is revealed by investigating earlier stages of development. Seven-day-old wildtype seedlings usually have six to eight leaves that are enclosed in the coleoptile. In cross sections at 2 to $3 \mathrm{~mm}$ above the coleoptilar node, the blades of the three oldest leaves (L1 to L3) are visible. At this developmental stage, Lls1 transcripts are expressed at low levels in all cells of leaves and coleoptile (Fig. 6). There is no preferential expression in the epidermis or other cell types, such as vascular bundles and sclerenchyma cells, which have just begun to differentiate at this stage, and no down-regulation in phloem precursors. In summary, in early leaf development (7-day-old seedlings) Lls 1 is expressed uniformly throughout all cells of leaves and coleoptile. This is unlike later in development (12- to 14-day-old seedlings, V4 stage) described above, when Lls 1 expression is up-regulated in the epidermis, sclerenchyma, protoxylem, and bundlesheath cells, and becomes undetectable in phloem.

The $l l s l$ mutant (reference allele) is caused by a $M u$ transposon insertion in the first exon of the gene (Gray et al. 1997). The expression of $L l s 1$ was analyzed in leaves 2 and 4 of this lls 1 mutant at 10 to 14 days after planting. At this stage, the tip of leaf 2 shows lesions, while leaf 4 has no lesions. By in situ hybridization, Lls1 transcript was detected in both of these leaves, showing that the loss-of-function phenotype of the $l l s 1$ mutant is not a result of abolished transcription. The pattern of Llsl expression in leaves of the llsl mutant is essentially the same as in wild-type leaves (data not shown). Therefore, a change in mRNA expression pattern does not cause the phenotype in the reference allele. Presumably the mutator insertion disrupts translation of a functional product.

\section{DISCUSSION}

Disease resistance is often measured by the following three criteria: reduced lesion number, reduced presence of viable pathogen in the lesion, and restricted lesion size. The $l l s 1$ mutation has elevated resistance to $C$. heterostrophus by the 
first two criteria but not the third, as the lesions formed are usually quite large. The $l l s 1$ mutant also resists $P$. sorghi in having reduced pustule number and size, but unlike for $C$. heterostrophus, the $P$. sorghi lesions produce pustules and are therefore fertile. There is also evidence that $l l s l$ has enhanced resistance to leaf lesion formation by Colletotrichum graminicola (causal agent of anthracnose), but little enhanced resistance to Cochliobolus carbonum (C. Simmons and S. Grant, unpublished data). The llsl enhanced disease resistance, like the $l l s 1$ lesion mimicry, is recessive when compared with heterozygous wild types. That the heterozygotes have some enhanced resistance relative to wild-type homozygotes was not directly addressed by this study, but comparisons to F2 wildtype homozygotes and inbred lines indicate that any such enhanced resistance would be small.

The lesion sterility and the reduced lesion number differ in their dependence upon light and maturity. The lesion sterility appears to result from the formation of the $l l s 1$-type necrotic lesions, the same sort of lesions that define the llsl lesion mimicry. The formation of these lesions is dependent upon both maturity and light. A previous study showed that red light $(650$ to $700 \mathrm{~nm}$ ) triggers spontaneous $l l s l$ lesion formation (Close et al. 1995). This study indicates that pathogen-induced $l l s l$-type lesion formation is not dependent upon the initial pathogen contact or initial plant response, because the whorl inoculation and dark inoculation experiments indicate that existing $C$. heterostrophus-like lesions become $l l s 1$-type after being shifted to the proper maturity zone or to light. The $l l s l$-type lesions expand to a large size and emanate a gradient of defense system activation, as indicated by markers of defense protein accumulation such as PR1. The combination of expanding necrosis and defense system activation is likely antimicrobial and accounts for the sterile $l l s l$-type lesions. Indeed, these lesions are also phytotoxic, for they eventually kill the plant. It remains unclear to what additional extent the physiological mechanism for generating necrosis and sterility in $l l s l$ lesions relates to that for normal defense reactions, such as sterile resistant lesions resulting from an incompatible hypersensitive response.

The reduced lesion number, on the other hand, does not appear to be dependent upon either maturity or light in the manner of $l l s l$-type lesion sterility. The whorl inoculation experiment indicates that the leaf tissue is resistant before it is competent to express $l l s 1$-type lesions. With $24 \mathrm{~h}$ of darkness prior to and 48 $\mathrm{h}$ after inoculation, the reduced lesion number is observed, suggesting it is light independent. Attempts to lengthen the pre- or post-inoculation darkness treatments were not possible because both wild-type and $l l s 1$ plants were stressed, and the resulting lesions were abnormally large, poorly delimited, and difficult to quantify. The possibility remains that light is necessary for the production of an antifungal state that persists in the darkness. However, it seems unlikely that it would be due to a lightdependent radical, as they are usually short-lived.

Formation of $l l s l$-type lesions does not itself appear to cause reduced lesion numbers following infection because it is observed both in the whorl inoculation before $l l s l$-type lesions appear and in the dark where $l l s 1$-type lesions seldom occur. The reduced lesion numbers are also not associated at the time of inoculation with markedly elevated PR proteins that might indicate a general defense system activation. It is possible that reduced lesion numbers result from the activation of defense systems, such as PR proteins, by the first-to-form $l l s 1$-type lesions after inoculation, thereby elevating resistance to subsequent lesion formation by other, slower growing fungal spores lying between these $l l s l$ lesions. This again seems unlikely because the reduced lesion numbers are observed in the whorl and dark inoculations where $l l s l$-type lesions are rare. It remains possible that other untested defense-related proteins or metabolites are constitutively elevated in $l l s 1$ or that they are poised to respond more rapidly upon inoculation than in wild-type plants. The reduced lesion number is associated with reduced fungal growth and infection rates at the leaf epidermis. The cause of this epidermal fungal toxicity is unknown, but should guide further study.

The reason for dependence on maturity for llsl-type lesion formation is unknown. Maize development is known to undergo a phase change from juvenile to adult tissues. This phase change is accompanied by changes in cell shape, epicuticular wax formation, cellular physiology, and disease resistance (Poethig 1990). We have observed that the transition from juvenile high epicuticular wax often occurs in the middle of the sixth leaf, and that it sometimes corresponds to the transition of $C$. heterostrophus-like lesions to llsl-type. This is not always the case, however, and so it appears that the competency to express $l l s 1$-type lesions is not strictly related to the developmental phase change, at least as indicated by epicuticular wax.

P. sorghi is a biotrophic pathogen that principally enters through stomata, and colonizes the mesophyll (Bushnell and Roelfs 1984). In this study, we observed that $P$. sorghi spore growth is inhibited at the epidermis and pustule formation and size are reduced. Nonetheless, successful penetrations of the stomata by $P$. sorghi can result in spore-bearing pustules without triggering $l l s l$-type lesion formation. Necrotrophic pathogens like $C$. heterostrophus and C. carbonum, as well as wounding by needle pricking (not shown), trigger $l l s l$-type lesion formation, apparently due to the tissue damage they inflict. P. sorghi not only avoids this, but can even maintain green islands within $l l s l$-type necrotic tissue. This means that $P$. sorghi can override the physiological disturbance in $l l s 1$ that causes the formation of $l l s l$-type necrosis, presumably in a manner akin to its well-documented formation of green islands in senescing leaf tissue. Specifically how P. sorghi does this is unknown, but this behavior in $l l s 1$ suggests it may be associated with a general suppression of reactive chemical species. Cytokinins have been implicated in green island formation, but preliminary results indicate that cytokinins do not inhibit formation of $l l s l$-type necrosis. That mature rust pustules can eventually become initiation centers for $l l s 1$-type necrotic lesions may be because at a very late stage the fungus is no longer actively engaged with the plant in suppressing signals that otherwise would lead to llsl-type lesions.

The in situ localization of $L l s 1$ gene expression in wild-type plants demonstrates that in leaf cross sections Llsl transcripts are expressed highly in the epidermis, and especially the sclerenchyma cap cells, but expression in the mesophyll is low. It is at the epidermis where fungal spore germination and growth are retarded, and this inhibition presumably results in the reduced lesion numbers with $C$. heterostrophus and $P$. sorghi. The lack of Lls1 expression in the mesophyll correlates with the site of successful colonization by $P$. sorghi. It appears therefore that in the epidermis of $l l s 1$ an antifungal state is produced, coinciding with where the Llsl gene is normally expressed. It is tempting to speculate that this is due to either 
the substrate for the Lls 1 enzyme or a metabolite resulting from it. This result also indicates a value to relating the particular site of gene expression in an organ such as a leaf to the strategies employed by pathogens for infecting it in order to better understand the resistance mechanism.

Loss of Lls1 function causes disease lesion mimicry and resistance. It is not known whether the normal function of $L l s 1$ is related to pathogen defense. That is, the wild-type gene function may not directly relate to pathogen defense, but when it is mutated pathogen resistance may result due to the accumulation of antimicrobial substances and/or activation of a defense pathway, possibly via reactive chemical species, some of which are known to activate plant defense systems. The Llsl gene product may be a suppresser of cell death in that it removes factors caused by cell injury that otherwise propagate cell death. Addressing the dependence of $l l s l$-type lesion formation on light and maturity seems pertinent to understanding the Lls1 gene's normal function, as does its developmental expression pattern revealed by the in situ studies. A model for Lls 1 function has been proposed in which lesion mimicry results from a failure of $L l s 1$ to alter a phenolic-based reactive species, likely light activated, thereby leading to activation of cell death pathways and lesion formation (Gray et al. 1997). Considering that $l l s 1$ also causes reduced lesion number, it appears that in $l l s l$ the substrate for the Lls1 gene product somehow leads to microbial toxicity without direct involvement of light or cell death.

\section{MATERIALS AND METHODS}

\section{Seed sources and growth conditions.}

The $l l s 1$ allele used was the reference allele obtained from the Maize Genetics Cooperative, University of Illinois, Urbana/Champaign. Seed populations were segregating 1:1 for lls 1 and wild-type heterozygotes, and were produced from a cross of $l l s 1 / l l s 1$ pollen by $l l s 1 / L l s 1$ wild-type heterozygote ears. Throughout this paper wild-type or wild-type sibling generally refer to a wild-type heterozygote sibling from the 1:1 segregating population unless otherwise indicated. Seeds were planted in $8.89-\mathrm{cm}$ pots in Strong-Lite Universal Mix potting soil (Universal Mix, Pine Bluff, AZ) and grown in a greenhouse (16-h day, 20.6 to $34.8^{\circ} \mathrm{C}, 50 \%$ average relative humidity, 560 to $620 \mu \mathrm{E} \mathrm{s}^{-1} \mathrm{~m}^{-2}$ of light from both sun and halogen lamps, averaging 25 heat units per day). Plants were grown for 14 to 18 days until the V5 stage (approximately 25 $\mathrm{cm}$ height) and inoculated.

For the in situ hybridization experiments, the $l l s 1$ seed described above and wild-type seeds for Mo17 and B73 inbreds were grown in the greenhouse to the V4 stage, approximately 2 weeks. At this stage spontaneous $l l s 1$-type lesions occurred on leaf 1 and the tip of leaf 2, but not on leaves 3 or 4 . Tissue for cross sections was taken from the tips of leaves 2 and 4 , and the middle of leaf 4 , for both $l l s 1$ and wild-type inbreds. For the vegetative apices sections, seedlings were grown for 7

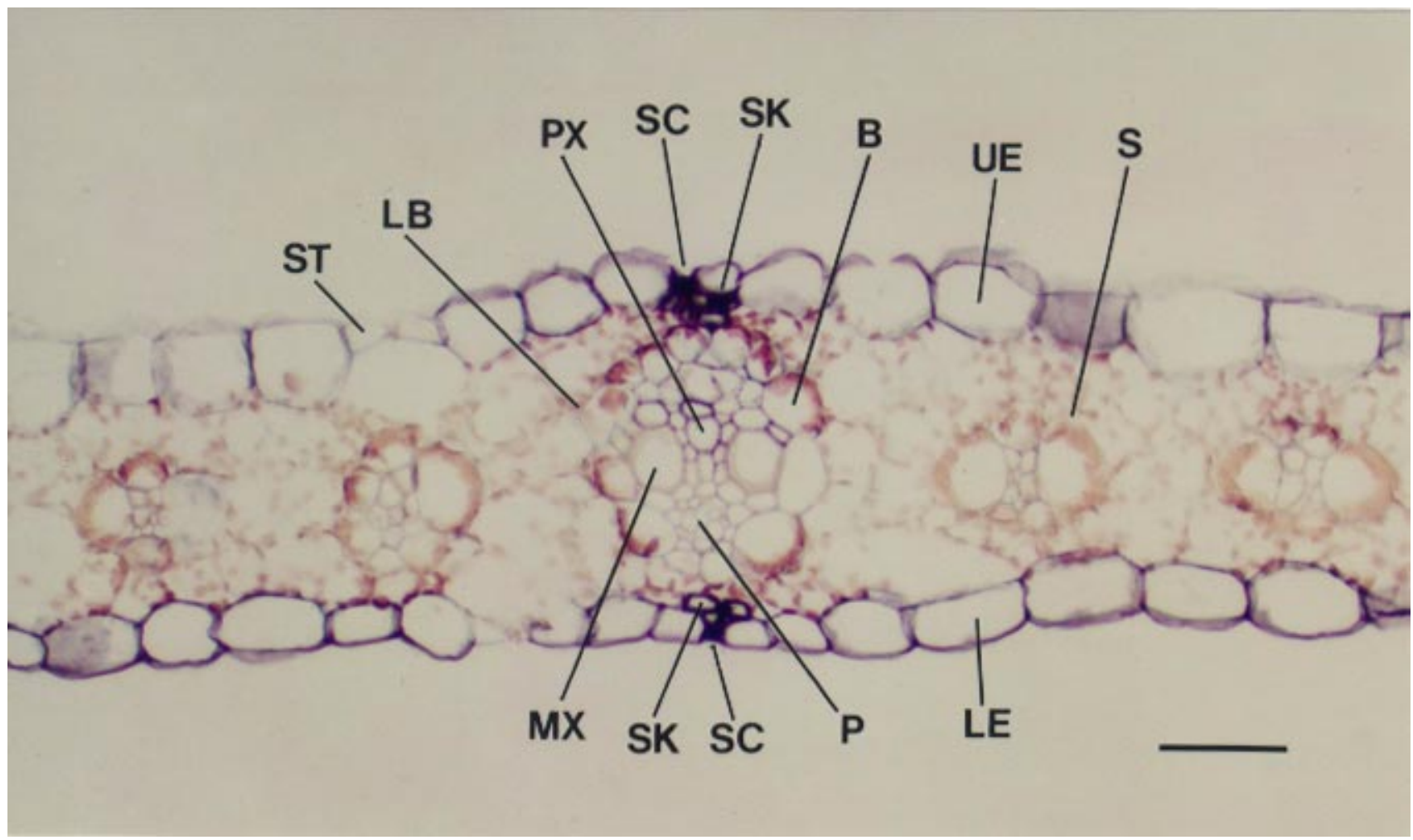

Fig. 5. In situ localization of $L l s 1$ transcript in wild-type leaves. Sections were probed with digoxigenin-labeled Lls1 RNA probe; hybridization to intracellular mRNA is detected via antibody-mediated color reaction. Strong expression signal appears blue, indicating high levels of transcript; weaker signal is brownish. Cross sections through the blade of a wild-type (Mo17) leaf (L2) at leaf tip. Plants were grown to V4 stage, approximately 2 weeks. Strong Llsl expression signal is detected in upper and lower epidermis, including silica cells and stomates, and in sclerenchyma cells. Signal intensity is intermediate in protoxylem elements and bundlesheath cells, low in mesophyll cells. Expression is undetectable in metaxylem, phloem, and companion cells. Pattern is the same in all types of bundles: large, intermediate (not represented in this section), and small. Sense strand control probe does not give any significant background signal. B, bundlesheath; LB, large bundle; LE, lower epidermis; MX, metaxylem; P, phloem; PX, protoxylem; S, small bundle; SC, silica cell, SK, sclerenchyma; ST, stomate; UE, upper epidermis. Bar $=50 \mu \mathrm{m}$. 
days, and $0.5-$ to $1.0-\mathrm{cm}$ segments constituting the coleoptilar node were harvested.

\section{Pathogen sources and inoculation conditions.}

Cochliobolus heterostrophus (Drechs.) Drechs. race 0 (anamorph: Bipolaris maydis; causal agent of southern corn leaf blight) isolate TX001, was obtained from field sources at Pioneer Hi-Bred and maintained on potato dextrose agar medium or for long-term storage in silica gel as described (Dhingra and Sinclair 1995). Puccinia sorghi Schwein. (causal agent of common rust) isolate PSO001, was obtained from field sources at Pioneer Hi-Bred and maintained on B73 inbred seedling leaves essentially as described (Dhingra and Sinclair 1995). For general leaf inoculation, spore suspensions of $4 \times 10^{4}$ per $\mathrm{ml}$ of $0.02 \%$ Tween 20 were sprayed as an aerosol on the leaves. Approximately $0.5 \mathrm{ml}$ was applied per leaf late in the afternoon.

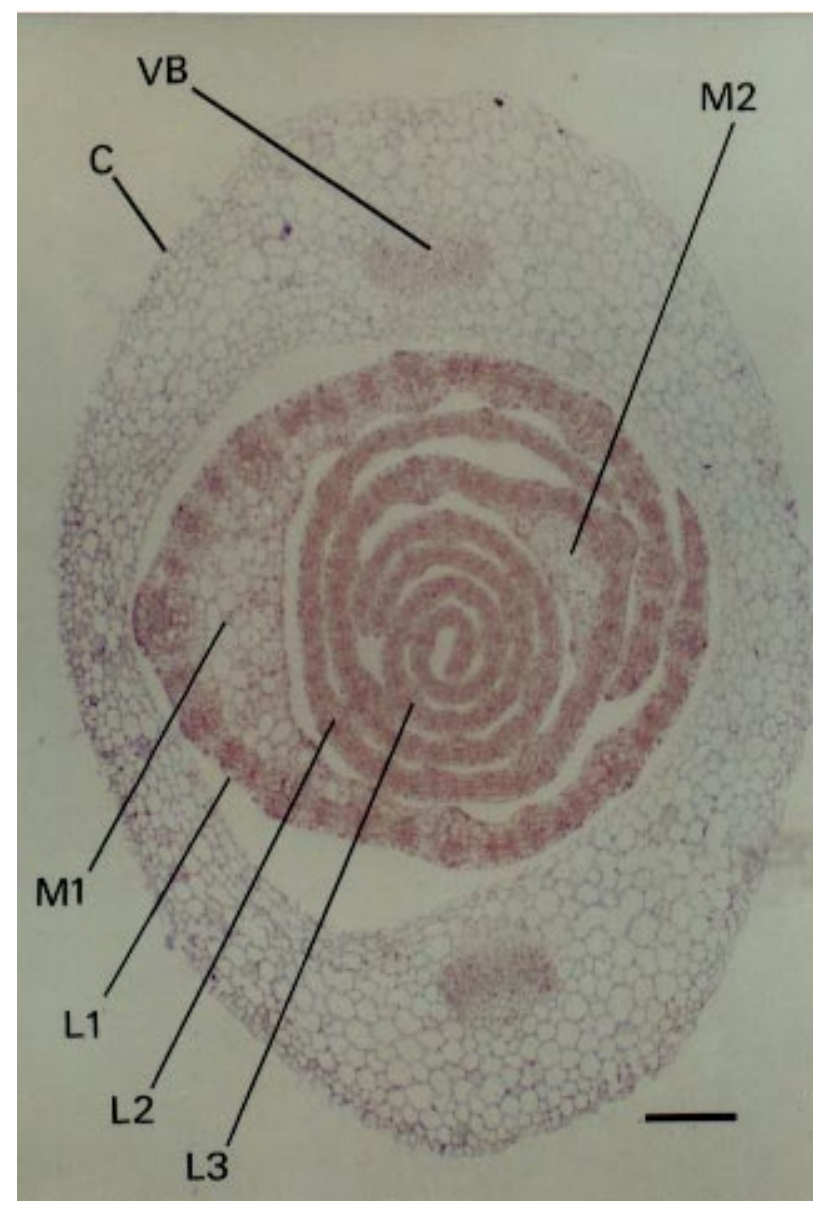

Fig. 6. In situ localization of Lls1 transcript in wild-type seedlings. Cross section through coleoptile and blades of three oldest leaves (L1L3) at about $3 \mathrm{~mm}$ above the coleoptilar node of a wild-type seedling (B73). Plants were grown for 7 days in the dark. At this stage, seedlings have a total of about seven leaves, none of which have emerged from the coleoptile. A low level of Lls1 expression is detected in all cells of young leaves and coleoptile, as evidenced by the brownish color. In contrast to later stages of development shown in Figure 5, expression is not elevated in epidermis or other cell types. Likewise, vascular bundles of the coleoptile do not show differential Lls1 expression, as observed in vascular bundles of leaves at later developmental stages. C, coleoptile, L1-3, leaves 1-3; M1 and M2, midribs of leaves 1 and 2, respectively; $\mathrm{VB}$, vascular bundle of coleoptile. $\mathrm{Bar}=200 \mu \mathrm{m}$.
The plants were then immediately covered with a plastic tent and kept at room temperature in order to enhance humidity and spore germination. The plastic tent was removed early in the morning, and the plants were returned to the greenhouse for the duration of the experiment. For whorl inoculation with $C$. heterostrophus, $0.2 \mathrm{ml}$ of $4 \times 10^{4}$ spores per $\mathrm{ml}$ of $0.02 \%$ Tween 20 was deposited in the whorl. After the rust inoculation, the plants were moved to a growth chamber (14-h day, $27^{\circ} \mathrm{C}, 80$ to $90 \%$ relative humidity, 200 to $300 \mu \mathrm{E} \mathrm{s}^{-1} \mathrm{~m}^{-2}$ from both fluorescent and incandescent lamps) to avoid rust contamination of the greenhouse. These less bright conditions slowed, but did not stop, the progression of $l l s l$-type lesions that otherwise turn the $l l s l$ leaves nearly fully necrotic and thereby preclude pustule formation 7 to 10 days after inoculation.

\section{Data collection and observation.}

The plants were kept in the greenhouse up to $48 \mathrm{~h}$ after inoculation, and then the lesions were counted, photographed, and investigated for viable fungal presence. Usually $48 \mathrm{~h}$ after $C$. heterostrophus pathogen inoculation the lesions were counted. The rust pustules were counted 7 to 10 days after inoculation. Leaf photographs were taken with an MP4 camera mount, a 35$\mathrm{mm}$ camera, and Kodak 160T film. Progression of spore growth and lesion formation was observed $48 \mathrm{~h}$ after inoculation with $C$. heterostrophus, and 3 to 4 days after $P$. sorghi inoculation, with a Nikon compound microscope at $\times 40$ and $\times 100$ magnifications. To enhance the visibility of the fungal hyphae, the leaves were stained with $10 \%$ phenol, $10 \%$ lactic acid, $20 \%$ glycerin, $0.035 \%$ aniline blue. Microscope photographs were taken with a 35-mm camera and Kodak 100 ASA film.

To determine whether viable fungal pathogens existed in the lesions, 3 to 8 inoculated leaves per treatment were surface sterilized in $70 \%$ ethanol for $2 \mathrm{~min}$, followed by three washes with sterile water. Under sterile conditions, 6 to 15 triangular wedges ( 2 to $3 \mathrm{~mm}$ on a side) from as many lesions were cut out per leaf such that one triangle point included a lesion margin. These leaf wedges were placed on carrot agar medium (broth from 3\% boiled diced carrots, $2 \%$ agar) and incubated at room temperature. Wedges yielding fungal outgrowth from the lesion tip were noted usually 48 to $72 \mathrm{~h}$ later. Plates were kept until fungal sporulation for positive identification of the recovered pathogen.

For Western analysis, leaf tissue was harvested 2 to 3 days after inoculation, frozen in liquid nitrogen, ground to a powder, and extracted with $50 \mathrm{mM}$ citric acid, $6 \mathrm{mM}$ sodium ascorbate, $50 \mathrm{mM} \mathrm{Na} \mathrm{HPO}_{4}, \mathrm{pH} 2.8$. Total protein levels were measured with the Bradford dye-binding assay. Equal protein amounts $(0.5$ $\mu \mathrm{g}$ ) were loaded in each lane of a 4 to $20 \%$ gradient polyacrylamide gel and electrophoresed. A positive control sample obtained from a different lesion mimic expressing high levels of PR proteins was included, as were molecular weight markers (Novex, San Diego, CA). Proteins were electro-eluted onto polyvinylidene difluoride (PVDF) membrane according to the manufacturer's instructions (Novex). The tobacco PR1b (GenBank accession no. X17680) and chitinase B (GenBank accession no. X64519) cDNAs were publicly obtained and overexpressed at Pioneer Hi-bred in Escherichia coli. For PR1b the pET28a expression vector was used (Novagen, Milwaukee, WI). The PR1b was expressed as inclusion bodies and those inclusion bodies were solubilized with $6 \mathrm{M}$ urea. The solubilized inclusion bodies were then loaded onto a TALON metal affinity resin column (Clonetech Laboratories, Palo Alto, CA) 
and refolded on the column in $50 \mathrm{mM}$ Tris, pH8.0, $500 \mathrm{mM}$ $\mathrm{NaCl}$. The refolded protein was eluted from the TALON column with $100 \mathrm{mM}$ imidazol. For chitinase B the expression vector was pGEX4T (Pharmacia Biotech, Piscataway, NJ). The protein was purified by affinity column chromatography with glutathione Sepharose $4 \mathrm{~B}$, and protein liberation by thrombin cleavage according to the manufacturer's recommendations (Pharmacia Biotech). The antiserum was prepared in rabbits and used in 1:1,000 to 1:5,000 dilutions for probing the Western blots. The secondary antibodies and chemi-luminescence detection system were used according to the manufacturer's recommendations (Tropix, Bedford, MA).

\section{In situ localization of $L l s 1$ gene expression.}

Leaf tissue was fixed in FAA (50\% ethanol, $10 \%$ formalin, $5 \%$ acetic acid). All other plant material was fixed in $4 \%$ paraformaldehyde in PBS (10 mM Na-phosphate $\mathrm{pH} 7.5,150$ $\mathrm{mM} \mathrm{NaCl}$ ) overnight at $4^{\circ} \mathrm{C}$. After being fixed, tissues were dehydrated in a series of ethanol and Histoclear (National Diagnostics, Atlanta, GA) and embedded in paraffin wax (ParaplastPlus; Fisher Scientific, Pittsburgh, PA). Tissue sections $(8$ to $10 \mu \mathrm{m}$ ) were cut on an Olympus microtome (CUT4060) and mounted on ProbeOn Plus microscope slides (Fisher Scientific). Tissue fixation and in situ hybridization were carried out as described (Jackson 1991)

An 0.8-kb NotI-PstI fragment representing the central portion of the Lls1 gene (Gray et al. 1997) was subcloned into Bluescript pBSIIKS(+) vector, linearized, and used as template for the production of riboprobes. Antisense and sense riboprobes were labeled with digoxigenin-11-UTP by in vitro transcription with T7 or T3 RNA polymerase (Boehringer Mannheim, Mannheim, Germany); reactions were carried out according to the manufacturer's protocol. Probes were purified and hydrolyzed as described (Jackson 1991). After immunological detection of RNA hybrids as described (Coen et al. 1990), slides were washed in water, dehydrated through an ethanol wash series, rinsed twice in Histoclear, and mounted in Merckoglas (EM Science, Gibbstown, NJ). Specimens were viewed with a Nikon Labophot-2 microscope under brightfield illumination. Photographs were taken with Kodak 200 ASA 35-mm color negative film.

\section{ACKNOWLEDGMENTS}

We thank Nasser Yalpani, Dan Altier, and Jon Duvick for helpful advice and comments. We also thank Tony Nevshemal, Lisa Marshall, and Valerie Sondag for assisting with the Western analysis, Dick Christensen and crew for managing the plants in the greenhouse, and Barb Fleener, Cara Jensen, Jackie Thompson, and Kelly Searcy for assisting with the fungal pathogen preparations.

\section{LITERATURE CITED}

Asada, K. 1992. Production and scavenging of active oxygen in chloroplasts. Pages 173-192 in: Molecular Biology of Free Radical Scavenging Systems. J. G. Scandalios, ed. Cold Spring Harbor Laboratory, Cold Spring Harbor, NY.

Bushnell, W. R., and Roelfs, A. P., eds. 1984. The Cereal Rusts. Vol. 1: Origins, Specificity, Structure, and Physiology. Academic Press, Orlando, FL.

Close, P. S., Gray, J., and Johal, G. 1995. Observations of the effect of light on the progression of lethal leaf spot1 mutations. Maize Genet. Newsl. 69:48-49.

Coen, E. S., Romero, J. M., Doyle, S., Elliott, R., Murphy, G., and Carpenter, R. 1990. Floricaula, a homeotic gene required for flower de- velopment in Antirrhinum majus. Cell 63:1311-1322.

Dangl, J. L, Dietrich, R. A., and Richberg, M. H. 1996. Death don't have no mercy: Cell death program in plant-microbe interactions. Plant Cell 8:1793-1807.

Dhingra, O. D., and Sinclair, J. B. 1995. Basic Plant Pathology Methods. 2nd ed. Lewis Publishers, Boca Raton, FL.

Dietrich, R. A., Delaney, T. P., Uknes, S. J., Ward, E. R., Ryals, J. A., and Dangl, J. L. 1994. Arabidopsis mutants simulating disease resistance response. Cell 77:565-577.

Dixon, R. A., and Paiva, N. L. 1995. Stress-induced phenylpropanoid metabolism. Plant Cell 7:1085-1097.

Gray, J., Close, P. S., Briggs, S. P., and Johal, G. S. 1997. A novel suppresser of cell death in plants encoded by the Lls1 gene of maize. Cell 89:25-31.

Greenberg, J. T., and Ausubel, F. M. 1993. Arabidopsis mutants compromised for the control of cellular damage during pathogenesis and aging. Plant J. 4:327-341.

Hammond-Kosack, K. E., and Jones, J. D. G. 1996. Resistance genedependent plant defense responses. Plant Cell 8:1773-1791.

Herbers, K., Meuwly, P., Frommer, W. B., Metraux, J.-P., and Sonnewald, U. 1996. Systemic acquired resistance mediated by the ectopic expression of invertase: Possible hexose sensing in the secretory pathway. Plant Cell 8:793-803.

Hockenbery, D. M., Oltvai, Z. N., Yin, X.-M., Milliman, C. L., and Korsmeyer, S. J. 1993. Bcl-2 functions in an antioxidant pathway to prevent apoptosis. Cell 75:241-251.

Hoisington, D. A. 1985. Linkage studies of lesion and necrotic mutants. Maize Genet. Coop. News1. 58:52-84.

Hu, G., Richter, T. E., Hulbert, S. H., and Pryor, T. 1996. Disease lesion mimicry caused by mutations in the rust resistance gene rp1. Plant Cell 8:1367-1376.

Jabs, T., Dietrich, R. A., and Dangl, J. L. 1996. Initiation of runaway cell death in an Arabidopsis mutant by extracellular superoxide. Science 273:1853-1856.

Jackson, D. P. 1991. In situ hybridization in plants. Pages 163-174 in: Molecular Plant Pathology: A Practical Approach. D. J. Bowles, S. J. Gurr, and M. McPhereson, eds. Oxford University Press, Oxford.

Johal, G. S., Hulbert, S., and Briggs, S. P. 1995. Disease lesion mimics mutations in maize: A model for cell death in plants. BioEssays 17: 685-692.

Johal, G. S., Lee, E. A., Close, P. S., Coe, E. H., Neuffer, M. G., and Briggs, S. P. 1994. A tale of two mimics: Transposon mutagenesis and characterization of two disease lesion mimic mutations in maize. Maydica 39:69-76.

Kane, D. J. Sarafin, T., Auton, S., Hahn, H., Gralla, F., Valentine, J., Ord, T., and Bredesen, D. 1993. Bcl-2 inhibition of neural death: Decreased generation of reactive oxygen species. Science 262:1274-1277.

Low, P. S., and Merida, J. R. 1996. The oxidative burst in plant defense: Function and signal transduction. Physiol. Plant. 96:533-542.

Neuffer, M. G., Coe, E. H., and Wessler, S. R., eds. 1997. Mutants of Maize. Cold Spring Harbor Laboratory Press, Plainview, NY.

Nicholson, R. L., and Hammerschmidt, R. 1992. Phenolic compounds and their role in disease resistance. Annu. Rev. Phytopathol. 30:369-389.

Obanni, M., Hipskind, J., Tsai, C. Y., Nicholson, R. L., and Dunkle, L. D. 1994. Phenylpropanoid accumulation and symptom expression in the lethal leaf spot mutant of maize. Physiol. Mol. Plant Pathol. 44: 379-388.

Poethig, R. S. 1990. Phase change and the regulation of shoot morphogenesis in plants. Science 250:923-930.

Pryor, T. 1987. The origin and structure of fungal disease resistance genes in plants. Trends Genet. 3:157-161.

Tenhaken, R., Levine, A., Brisson, L. F., Dixon, R. A., and Lamb, C. 1995. Function of the oxidative burst in hypersensitive disease resistance. Proc. Natl. Acad. Sci. USA 92:4158-4163.

Ullstrup, A. J., and Troyer, A. F. 1967. A lethal leaf spot of maize. Phytopathology 57:1282-1283.

Walbot, V., Hoisington, D. A., and Neuffer, M. G. 1983. Disease lesion mimic mutations. Pages 431-442 in: Genetic Engineering of Plants. T. Kosuge, C. P. Meredith, and A. Hollaender, eds. Plenum Press, New York.

Wolter, M., Hollricher, K., Salamini, F., and Schulze-Lefert, P. 1993. The mlo resistance alleles to powdery mildew infection in barley trigger a developmentally controlled defence mimic phenotype. Mol. Gen. Genet. 239:122-128. 